\title{
An integrated TSP-GA with EOL cost model for selecting the best EOL option
}

\author{
Zakri Ghazalli and Atsuo Murata
}

Department of Intelligent Mechanical Systems, Division of Industrial Innovation Sciences, Okayama University, Tsushima Naka, 1-1-1, Kita-Ku, Okayama City, 700-8530, Japan

\begin{tabular}{|c|c|}
\hline A R T I C L E I N F O & A B S T R A C T \\
\hline $\begin{array}{l}\text { Article history: } \\
\text { Received } 11 \text { May } 2010 \\
\text { Received in revised form } \\
\text { June, } 20,2011 \\
\text { Accepted } 21 \text { June } 2011 \\
\text { Available online } \\
\text { 22 June } 2011 \\
\text { Keywords: } \\
\text { Disassembly sequence planning } \\
\text { Traveling salesman problem } \\
\text { Genetic algorithm } \\
\text { Environmental cost } \\
\text { Economical cost } \\
\text { End-of-life profit } \\
\text { Net present value }\end{array}$ & $\begin{array}{l}\text { This paper presents our research works on integrating disassembly sequence planning with cost } \\
\text { model for end-of-life (EOL) product. This paper has two objectives. The first objective is to } \\
\text { optimize disassembly sequence of the EOL product. We integrate a traveling salesman problem } \\
\text { approach with genetic algorithm in finding the optimal disassembly sequence for disassembling } \\
\text { the EOL product. Based on this optimal sequence, the second objective is to identify the best } \\
\text { EOL option. We employ EOL profits and net present value of parts and subassemblies of the } \\
\text { EOL product to determine the best EOL option of components and parts of the EOL product. } \\
\text { The predicted results showed that the developed cost model has reached a good correspondence } \\
\text { with the established methods. }\end{array}$ \\
\hline
\end{tabular}

\section{Introduction}

As the world population increases, the amount of waste generated at the end-of-life of products has become a severe problem in many countries. In response, producers devoted a considerable effort to demonstrate new methods and activities that reflect environmental consciousness in producing products. The rapid pace of technological changes within a short time renders some products obsolete even though they are still new and in an excellent condition. These products need to be processed in an environmentally benign and cost-effective manner. Most of the common practice is to treat these products with end-of-life (EOL) processes. These processes aim to minimize the amount of waste sent to landfills by recovering materials and parts of old or obsolete products by recycling and remanufacturing them (McGovern \& Gupta, 2007; Kaebernick et al., 2002; ElSayed et al., 2010). There are many attributes of a product that enhance product recovery. Examples of attributes include ease of disassembly, modularity, material used, efficient cross-industrial reuse of common parts and so on. Among these attributes, the crucial one is the disassembly (McGovern \& Gupta, 2007).

Disassembly is a methodical extraction of valuable parts/subassemblies and materials from discarded products through a series of operations. After disassembly, reusable parts or subassemblies are cleaned, refurbished, tested and directed to the part or subassembly inventory for remanufacturing

* Corresponding author. Tel:+8186-251-8056; Fax: +8186-251-8056

E-mail: zakri@iims.sys.okayama-u.ac.jp (Z. Ghazalli)

(C) 2011 Growing Science Ltd. All rights reserved. doi: $10.5267 /$ j.ijiec.2011.06.005 
operations. The recyclable materials can be sold to raw-material suppliers while the residuals are sent to landfills. A disassembly process is relatively an expensive one, due to its labour-intensive nature (Kongar \& Gupta, 2006). Hence, finding a feasible solution and an optimal or near optimal solution is desirable. Disassembly sequence planning (DSP) is one of the methods that can provide feasible disassembly sequences of an EOL product.

Several researchers have focused their studies on the disassembly planning system (e.g. Zussman et al., 1994; Penev \& De Ron, 1996; Xirouchakis \& Kritis, 1997; Srinivasan \& Gadh, 1998; Gungor \& Gupta, 2001; Lambert \& Gupta, 2005). However, these authors presented theoretical studies and did not demonstrate practical applications. On the other hand, Ke and Henrioud (1994), Henrioudet al.(1995) and Diniet al. (2001) presented their works on developing disassembly models based on graph-models such as adjacency graph, adjacency matrix, petri-net, AND/OR graph to represent product architecture, collect, and store relevant information of the EOL product. However, none of them discussed the optimization of the disassembly process. To cope with this limitation, we consider the genetic algorithm (GA) approach to find the optimal disassembly sequence.

Genetic algorithm has been gaining its popularity as it can provide a quick and cost-effective solution in finding an optimal or sub-optimal disassembly sequence. It involves the activities of encoding a defined problem into chromosome, evaluating the performance of chromosome based on the defined function of fitness, selecting the most optimal disassembly sequence via the operations of crossover, mutation and reproduction of chromosome. Hui et al. (2008), Seo et al. (2002) and Li et al. (2005) employed GA to evaluate the optimal disassembly sequence. A penalty was assigned to the strings so that it can be eliminated in mutation process. However, the weakness of these approaches is that infeasible solution of disassembly sequence occurred during crossover and mutation process. To overcome this constraint, we incorporated a traveling salesman problem (TSP) method into GA.

TSP is a typical example of a non-deterministic polynomial time hard (NP-hard) problem. TSP aims at finding the shortest tour that passes through every city only once. In TSP, the disassembly task is modelled as a city that has to be travelled by a salesman. The most important task in TSP is to ensure that the salesman travels in a feasible route without violating the precedence relationship between disassembly operations. Hence, the TSP employed the precedence constraint to ensure that a salesman only travels to cities in a feasible sequence (Azab et al., 2011). Several researchers presented their works on precedent constraint. Kusiak and Finke (1987) presented a branch-andbound algorithm method for the scheduling problem. Renaud et al. (2000) applied a heuristic approach to solve pickup and delivery TSP. Seo et al. (2001) employed AND/OR graph to maintain the feasible disassembly sequence. However, neither of these approaches can present an optimal disassembly sequence for the problem. To cope with this limitation, we used a topological sort (TS) concept in a directed graph, the advantage of which is to enable us to generate a feasible disassembly sequence without requiring any modification for the initial population and offspring generated in the mutation process.

In determining EOL alternative selection, many literatures presented results on economics of disassembly, costs, and revenues of EOL. Das et al. (2000) introduced disassembly effort index (DEI) to measure the ease of disassembly. Ayres et al. (1997) employed a product gain approach to determine an EOL option. Krikke et al. (1998) employed a dynamic programming algorithm to determine an optimal EOL option. Tang et al. (2004) employed a net present value (NPV) which takes into account the time value of money to evaluate the economic value of disassembly strategies. Ritchey et al. (2005) developed a framework to evaluate the remanufacturing options from the economical point of view. Tan and Kumar (2008) and Xanthopoulus and Iakovou (2009) used linear programming models to evaluated EOL options. However, none of these authors has considered the benefits of evaluating EOL options from the environmental perspectives. To cope with these 
limitations, we employed EOL values which integrate economical costs with environmental costs in selecting the best EOL options.

Lee et al. (2001) incorporated both cost and environmental impact estimation into EOL disassembly chart model. Staikos and Rahimifard (2007) integrated AHP with benefit-cost analysis to find the most suitable EOL option. The disadvantage of these methods is that it requires even experienced decision makers to understand the problem and deal with the complexity of environmental assessment in finding the best EOL strategy. To overcome these limitations, we employed an EOL profit approach, which consider economical, environmental, and disassembly costs in finding the best EOL option. In summary, although many researchers presented their works on disassembly planning, few studies have been done on integrating an optimization tool such as GA of disassembly planning with economical and environmental cost factors for selecting the best EOL option of parts and subassemblies of an EOL product. Our address of disassembly optimization problem can be defined as follows: Given an EOL product, determine the optimal disassembly sequence in order to:

(1) Maximize the net revenue resulting from the benefits accrued from the recovery of parts and subassemblies, and

(2) Minimize the costs incurred while disassembling the product or its components.

Realizing the importance of implementing an optimal disassembly and a cost-effective recovery of the EOL product after the end of its useful life, we extended the Stage II: part of selecting an EOL of parts and components presented by Ghazalli and Murata (2011). In this extended work, we utilized the TSP-GA as an approach for determining the optimal disassembly sequence. Based on this sequence, we evaluate the selection of EOL options through the maximized net revenue (EOL profit).

This paper is organised as follows. Section 2 presents an overview of the integrated TSP-GA with the EOL cost model. Section 3 presents the example of the application of the proposed method. In this section, the results of TSP-GA are compared with those of the established methods of Moon et al. (2002) and Kongar and Gupta (2006).Additionally, the outcomes for selecting the best EOL options are corroborated with the works of Johnson (2002) and Ghazalli and Murata (2011).In Section 4, we discuss the results. Finally, in Section 5, we draw conclusions and highlight directions for further research.

\section{Integration of the TSP-GA for disassembly sequencing method}

\subsection{Overview of the integrated approach}

The integrated approach is categorized into three types of assessments. The first assessment is aimed at finding the optimal disassembly sequence of product EOL using TSP-GA. The second and third assessments are aimed at finding the EOL value of parts and subassemblies of EOL product. Then, based on the integration of disassembly, economical and environmental costing, the EOL profits and net present values are assessed by finding the EOL options of parts and subassemblies of the EOL product. The outline of the integrated method is illustrated in Fig.1. As shown in Fig. 1, we begin the evaluation process by setting up the bill of material (BOM) of the parts and subassemblies of the product EOL. The BOM consists of the disassembly, economic and environment parameters. These parameters are used to identify the optimal disassembly sequence of an identified EOL product and to select the best EOL option for parts and subassemblies of the EOL product.

In the first step, we gather the information for disassembly assessment of parts and subassemblies of the EOL product. This information includes the disassembly time, tasks, orientations, tool used to disassemble parts and subassemblies and precedent constraints between parts to be disassembled. Next, we gather the information for economical and environmental assessments. This information includes the type of materials, labour rate, recycling rate, landfilling rate and so on. Details of 
information required for setting up the BOM are given in Table 1. After setting up the BOM, we start the evaluation for determining the optimal disassembly sequence. We employ an integrated traveling salesman problem with genetic algorithm (TSP-GA) approach to find an optimal disassembly sequence. In TSP-GA, parts and subassemblies of the EOL product have been considered as cities. Additionally, the time to disassemble a part or subassembly from its adjacent part or subassembly has been set as a traveling distance from one city to another. A precedence constraint is employed to ensure that the TSP-GA generates a feasible disassembly sequence. Then, the evaluation of fitness function, the processes of crossover, mutation, and reproduction were assigned to evaluate and find the minimum value of feasible disassembly sequence.

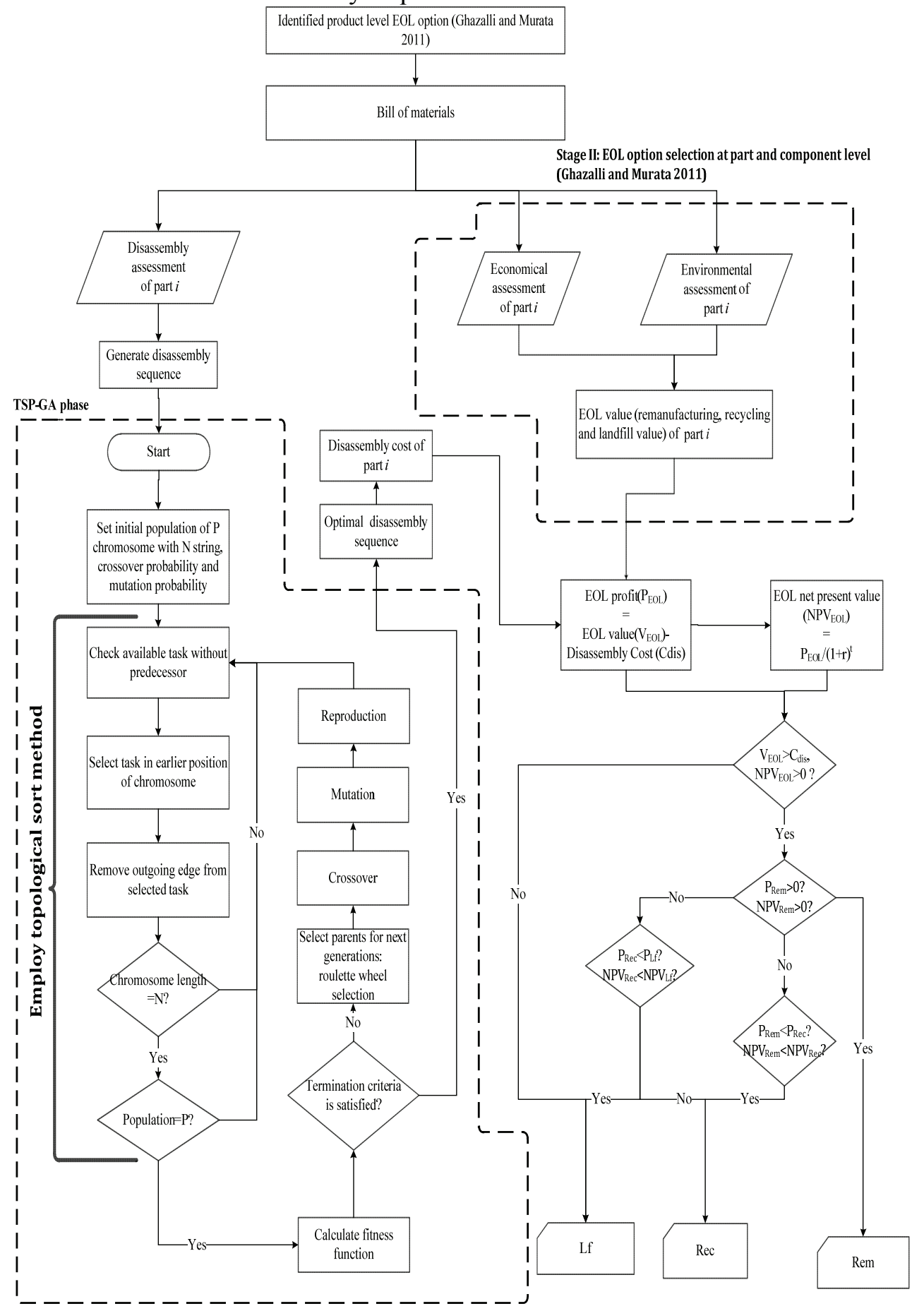

Fig.1. Overview of the integrated TSP-GA-EOL cost model approach 
After the feasible disassembly sequence is optimized, the optimal disassembly costs of parts and subassemblies of the EOL product are generated. On the other hand, based on the information in BOM (see Table 1), we assess the economical and environmental costs. After that, we evaluate the EOL values of each part and subassembly of the EOL product. Then, the EOL profit and net present value (NPV) are obtained. The differences between EOL values and disassembly costs (calculated based on the optimal disassembly sequence) produce the values of EOL profit, which are used to generate NPV for parts and subassemblies of the EOL product. If the values of EOL profit and NPV for remanufacturing are equal to or less than zero, the parts or subassemblies under consideration should not be remanufactured. The parts are evaluated for recycling or landfill. If the values of EOL profit and NPV for recycling are equal to or less than landfill value, the parts or subassemblies are recommended for landfill. On the other hand, if the values of EOL profits and NPV for remanufacturing and recycling are equal to or less than zero, the parts or subassemblies should be considered for landfill.

\section{Table 1}

Required information for determining the best EOL option

\begin{tabular}{lll}
\hline No. & Required information & unit \\
\hline 1 & Part material & $\mathrm{kg}$ \\
2 & part weight & $\$$ \\
3 & cost of new part & \\
4 & optimal disassembly sequence & $\$$ \\
5 & disassembly time & $\$$ \\
6 & sorting time & $\$$ \\
7 & cleaning time & $\$$ \\
8 & refurbish time & $\$$ \\
9 & testing time & $\$$ \\
10 & assembly time & $\$$ \\
11 & labour cost & $\$$ \\
12 & transport cost & $\$$ \\
13 & storage cost & $\$$ \\
\hline
\end{tabular}

\subsection{Disassembly assessment for determine the optimal disassembly sequence}

To find an optimal sequence, we employ an integrated approach of TSP-GA. It comprises the following steps:

Generate numbers of population chromosome $(\mathrm{P})$ with the number of disassembly task $(\mathrm{N})$, Identify the feasible routes for TSP, Evaluate the function of fitness of each chromosome, Select chromosomes through roulette wheel selection method, and Implement crossover, mutation and reproduction of offspring.

\subsubsection{Generating the number of population chromosome for TSP}

The initialization step consists of establishing initial chromosomes, probability of crossover and mutation. We employed a random permutation selection to generate the chromosomes. The integer from Task 1 to Task $\mathrm{N}$ is generated in random sequence. These chromosomes depend on the number of population $(P)$. The size of population, probability of crossover $(P c)$ and mutation $(P m)$ were set to $150,0.5$ and 0.2 , respectively.

\subsubsection{Identifying the feasible route of TSP}

The sequences generated in sub-section 2.2.1 normally do not satisfy the precedence constraints. Hence, a disassembly precedence constraint has been taken into account to ensure that sequences of disassembled parts of an EOL product (chromosomes) are in a feasible order. The topological sort (TS) method has been employed to transform the infeasible sequence of these chromosomes into 
feasible ones. The TS is an ordering of disassembly sequence in a directed graph of a precedence diagram. An example of direct graph of a precedence diagram is illustrated in Fig. 2. The steps of TS for generating disassembly precedence sequence comprises investigating the available tasks of disassembly without a predecessor, selecting the disassembly task in earlier position and finally removing the edges from selected tasks.
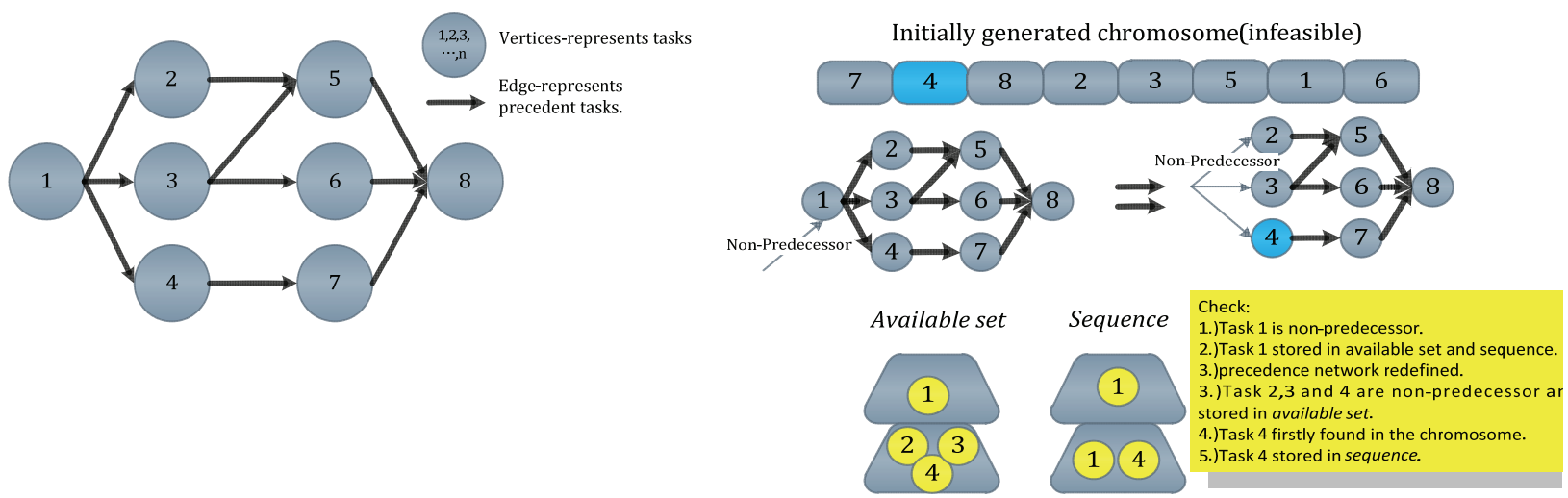

Fig.2. An example of precedence constraint diagram

Fig.3. Application of topological sort for obtaining a feasible sequence

\subsubsection{Investigating the disassembly tasks without predecessor}

Assume that the structure of an initial generated chromosome is in the order of Task 7, Task 4, Task

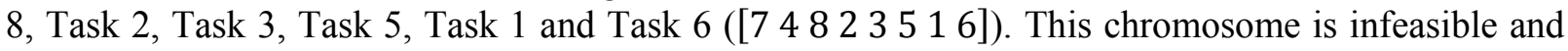
does not satisfy the precedence constraint as shown in Fig.3. In order to obtain the feasible solution, tasks without predecessor must be selected and stored in the available set (see Fig.3). For example, Task1 is the only task without a predecessor. Hence, it has been selected and stored in sequence.

\subsubsection{Selecting tasks in earlier position}

After the Task 1 has been stored in sequence, the Task 2, Task 3 and Task 4 are classified as the tasks without predecessor. We repeat the step in sub-section 2.2.2.1 by storing these tasks into availableset (see Fig. 3). Additionally, from these tasks, the Task 4 is the first to be found in the chromosome of

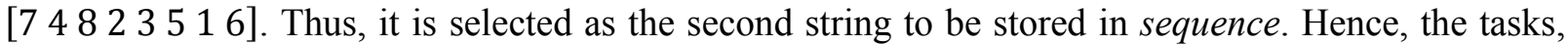
which have been stored in sequence for the second iteration, are the Task 1 and Task 4([14]).

\subsubsection{Removal of edge from the selected task}

After selecting Task 4, the outgoing edges from task 4 to task 7 are removed. The redefined availableset consists of Task 2, Task 3, and Task 7. The procedures from sub-section 2.2.2.1 to 2.2.2.3are repeated until the sequence reaches $N$.

\subsubsection{Calculation of function offitness}

The fitness function is performed based on the objective function. In this case, our aim is to minimize the total disassembly time. The objective function is represented as follows,

$f(x)=\min \sum_{i=1}^{n} \sum_{i=1}^{n}\left(t_{\text {disact }_{i j}}+t_{\text {dir }_{i j}}+t_{\text {dismt }_{i j}}\right) x_{i j}$ 


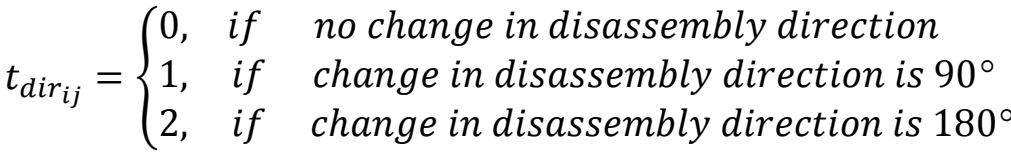

$t_{\text {dismethod }}$ ij $=\left\{\begin{array}{c}0, \text { if no change in disassembly method }(N) \\ 1, \text { if change in disassembly method }(D)\end{array}\right.$

$x_{i j}= \begin{cases}1, & \text { route } i \text { to } j \text { is in solution } \\ 0, & \text { otherwise }\end{cases}$

where $i$ and $j$ represent the disassembly task (node) from Task 1 to Task $N$, and $i \neq j$. The $t_{\text {disact }}{ }_{i j}$ is the time of the disassembly action. The $t_{d i r_{i j}}$ denotes the time penalty for each change of direction of node $i$ during disassembly in node $j$.

Table 2

Disassembly time, changes in disassembly method and direction of a desk phone body

\begin{tabular}{|c|c|c|c|c|}
\hline Part ID & Part Name & $t_{\text {disact }_{i j}}$ & Method & Direction \\
\hline 1 & Upper plastic casing & 3.8 & $\mathrm{~N}$ & $\mathrm{z}$ \\
\hline 2 & Speaker foam & 3.7 & $\mathrm{~N}$ & $\mathrm{z}$ \\
\hline 3 & 4 screws & 4.7 & $\mathrm{~N}$ & $\mathrm{y}$ \\
\hline 4 & Speaker & 3.7 & $\mathrm{~N}$ & $\mathrm{z}$ \\
\hline 5 & Plastic plate & 4.1 & $\mathrm{~N}$ & $\mathrm{z}$ \\
\hline 6 & PolyFlex circuit board 1 & 5.4 & $\mathrm{~N}$ & $\mathrm{z}$ \\
\hline 7 & PolyFlex circuit board 2 & 6.7 & $\mathrm{~N}$ & $\mathrm{z}$ \\
\hline 8 & screws & 4.5 & $\mathrm{~N}$ & $\mathrm{z}$ \\
\hline 9 & LCD panel\#1 - subassembly & 4.3 & $\mathrm{~N}$ & $\mathrm{z}$ \\
\hline 10 & Main circuit board & 4.3 & $\mathrm{~N}$ & $\mathrm{z}$ \\
\hline 11 & Elastometer key pad \#1 & 3.8 & $\mathrm{~N}$ & $\mathrm{z}$ \\
\hline 12 & Elastometer key pad \#2 & 3.8 & $\mathrm{~N}$ & $\mathrm{z}$ \\
\hline 13 & Elastometer key pad \#3 & 3.8 & $\mathrm{~N}$ & $\mathrm{z}$ \\
\hline 14 & Shift button & 3.7 & $\mathrm{~N}$ & z- \\
\hline 15 & 12 memory keys & 3.7 & $\mathrm{~N}$ & z- \\
\hline 16 & 3 display buttons & 3.7 & $\mathrm{~N}$ & z- \\
\hline 17 & Feature button & 3.7 & $\mathrm{~N}$ & z- \\
\hline 18 & Release button & 3.7 & $\mathrm{~N}$ & z- \\
\hline 19 & Hold button & 3.7 & $\mathrm{~N}$ & z- \\
\hline 20 & 12 dial keys & 3.7 & $\mathrm{~N}$ & z- \\
\hline 21 & 12 line keys & 3.7 & $\mathrm{~N}$ & z- \\
\hline 22 & Volume control & 3.7 & $\mathrm{~N}$ & z- \\
\hline 23 & Display cover - LCD\#1 & 6.1 & $\mathrm{~N}$ & $\mathrm{z}$ \\
\hline 24 & Display cover - LCD\#2 & 6.1 & $\mathrm{~N}$ & $\mathrm{z}$ \\
\hline 25 & LCD panel \# 2 & 4.3 & $\mathrm{~N}$ & $\mathrm{z}$ \\
\hline 26 & Hang up lever & 5.3 & $\mathrm{~N}$ & $\mathrm{z}$ \\
\hline 27 & Metal clip & 5.2 & $\mathrm{~N}$ & $\mathrm{z}$ \\
\hline 28 & Lower plastic casing & 3.8 & $\mathrm{~N}$ & $\mathrm{z}$ \\
\hline 29 & Lower plastic casing screws & 4.7 & $\mathrm{~N}$ & $\mathrm{z}$ \\
\hline 30 & 2 rubber pads & 5.5 & $\mathrm{~N}$ & $\mathrm{z}$ \\
\hline 31 & Plastic stand & 5.6 & $\mathrm{~N}$ & $x^{+}$ \\
\hline 32 & 2 rubber pads & 5.5 & $\mathrm{~N}$ & $\mathrm{z}$ \\
\hline 33 & PCB-Speaker Wire & 5.3 & $\mathrm{D}$ & $\mathrm{z}$ \\
\hline 34 & PCB-microphone wire & 5.3 & $\mathrm{D}$ & $\mathrm{z}$ \\
\hline 35 & Microphone & 3.6 & $\mathrm{~N}$ & $\mathrm{y}$ \\
\hline 36 & Microphone cover & 3.8 & $\mathrm{~N}$ & $\mathrm{z}$ \\
\hline
\end{tabular}


The $t_{\text {dismethod }}{ }_{i j}$ denotes the time penalty for each change of disassembly method of node $i$ in node $j$. If the disassembly method is changing from non-destructive disassembly to destructive disassembly from node $i$ to node $j$, the disassembly sequence is penalized by one second. Otherwise, the disassembly sequence is not penalized. The $x_{i j}$ is a decision variable constraint which is to ensure that the node is entered and exit from node $i$ to node $j$ only once. The $t_{\text {disact }}{ }_{i j}$, disassembly method and disassembly direction for the desk phone body are given in Table 2 , respectively.

\subsubsection{Roulette wheel selection}

The roulette wheel selection method is used to select parent chromosomes for regenerating the next chromosome. In this method, parent chromosomes are selected with a probability that is proportional to the fitness values obtained from the $E q$. (1). The probabilities of selecting a parent can be seen as spinning a roulette wheel with the size of the segment for each parent being proportional to its fitness. For chromosomes that have a good performance of fitness, the chances to be selected are higher. The roulette wheel selection can be represented as follows (Xing et al., 2005;Razali and Geraghty, 2011):

$p_{i}=\frac{f_{i}}{\sum_{i=1}^{N} f_{i}^{\prime}}$

where $f_{i}$ is the fitness values of the parent chromosomes $1,2,3, \ldots, \mathrm{N}$. The $p_{i}$ represents the chances of selecting the parent chromosomes $i$.

\subsubsection{Crossover}

We applied the moon crossover method for generating the two new offspring (Moon et al. 2002). The procedure of moon crossover is depicted in Fig.4.

Procedure: Moon crossover

Begin

1: Initialization: osp $\leftarrow$ null, $k \leftarrow 0$

2: Select two random chromosomes $P_{a}$ and $P_{b}, p_{a}=g 1 g 2 \ldots g J$ and $p_{b}=q 1 q 2 \ldots q J$;

3: Select two genes from $p_{a}$ at random.

4: osp $\leftarrow$ the substring between giand gjselected from $p a$.

5: if the length of $o s p=J$ then end

$6:$ else $S u b_{-} p b \leftarrow$ the remaining substring from the deleting genes which arealready selected from $p a$

7: end if

8: while (length of $o s p \neq J$ ) do

9: if $i=1$ then $i=J+1$;

$10: i \leftarrow i-1$;

$11: k \leftarrow k+1, k=1,2, \ldots$, length of $s u b \_p b$;

12:if $g i \neq q k$, then $o s p=<o s p$, gi, $q k>$;

13:else $g i=q k$, then $o s p=<o s p$, $g i,>$;

14:else if $j=J$ then

15: $i \leftarrow i-1$;

16: $k \leftarrow k+1, k=1,2, \ldots$, length of $s u b_{-} p b$;

17:if $g i \neq q k$, then $o s p=<q k, g i, o s p>$;

18:else $g i=q k$, then $o s p=<g i, o s p>$;

19:else

20: $i \leftarrow i-1$;

$21: k \leftarrow k+1, k=1,2, \ldots$, length of $s u b_{-} p b$;

22:if $g i \neq q k$, then $o s p=<g i, o s p, q k>$;

23:else $g i=q k$, then $o s p=<g i$, osp $>$; 


\section{4:end if}

25:end while

26:end procedure

Fig.4. Procedure of Moon crossover (Moon et al., 2002)

According to Fig.4, the $o s p$ is the offspring that is generated from the crossover between the first parent of the chromosome $\left(P_{a}\right)$ and the $S u b \_p_{b}$. The $S u b \_p_{b}$ represents the offspring generated from the second parent of the chromosome $\left(P_{b}\right)$. It is the remaining sub-string by deleting genes, which are already selected in $P_{a}$. The $g_{i}$ and $q_{k}$ are the genes in the $P_{a}$ and the second chromosome $\left(P_{b}\right)$, respectively. The moon crossover begins with initializing the $o s p$ and the numbers of genes in $s u b \_p_{b}$. Step 2 involves selecting the chromosomes of $P_{a}$ and $P_{b}$ at random. Assume that these chromosomes are $P_{a}=\left[\begin{array}{lllllll}5 & 1 & 7 & 2 & 4 & 6 & 3\end{array}\right]$ and $P_{b}=\left[\begin{array}{lllllll}3 & 6 & 1 & 4 & 2 & 5 & 7\end{array}\right]$. Step 3 involves the selection of two genes from the $P_{a}$. Suppose that the selected genes are the $o s p=\left[\begin{array}{ll}7 & 2\end{array}\right]$.

Steps 5 through 7depict the procedure to produce the $S u b p_{b}$. In this example, we obtain the $S u b p_{b}=$ [ $\left.\begin{array}{lllll}3 & 6 & 1 & 4 & 5\end{array}\right]$ from $P_{b}$. The Steps 8 through 25 are the procedures to add the $g_{i}$ and $q_{k}$ after the substring $o s p$. After generated the $S u b p_{b}$, we select the $g_{2}=1$ and $q_{1}=3$ from $P_{a}$ and $P_{b}$, respectively because $i=3-1$ and $\mathrm{k}=0+1$.Also, $i$ and $k$ are the position of the offsprings in the $o s p$ and $s u b p_{b}$, respectively. Hence, the offspring becomes $o s p=\left[\begin{array}{llll}1 & 7 & 2 & 3\end{array}\right]$. We repeat this procedure by adding the $g_{1}$ and $q_{2}$ to the offspring, and it becomes $o s p=\left[\begin{array}{llllll}5 & 1 & 7 & 2 & 3 & 6\end{array}\right]$. Next, we select the $g_{7}=3$ and $q_{3}=1$ from $P_{a}$ and $P_{b}$. However, these genes have appeared in the $o s p$, so we cannot add these genes into the $o s p$. Finally, we picked $q_{7}=4$ and the offspring becomes $o s p=$ [5172364]. We can produce the second offspring $o s p=\left[\begin{array}{lllllll}3 & 6 & 1 & 4 & 5 & 7 & 2\end{array}\right]$ by using the same procedures (Moon et al., 2002).

\subsubsection{Mutation}

The mutation operator makes random change to one or more elements of the strings. The mutation is carried out with small probabilities (Seo et al., 2001). In this study, we employed a swap mutation to the chromosome (Moon et al., 2002). First, two strings in chromosome are selected randomly. After that, the position of the selected strings is swapped to create a new chromosome. The purpose of swapping string at random is to avoid local optimum (Moon et al., 2002).

\subsection{Economical and environmental assessment of parts and subassemblies of the EOL product}

\subsubsection{Overview the structure of the economical and environmental assessment}

In this stage, economical and environmental costs were included for selecting the EOL options of parts and subassemblies of an EOL product. Fig. 1 illustrated the model for selecting the best EOL option. The EOL values of remanufacturing, recycling and land filling can be represented as follows (Lee et al., 2001; Ghazalli \& Murata, 2011):

$V_{\text {Rem }}=C_{p i}-C_{\text {Rem }}-C_{\text {Misc }}$

$V_{\text {Rec }}=C_{\text {Rec }}-C_{\text {Misc }}$

$V_{L f}=-C_{L f}-C_{M i s c}$

$C_{\text {Misc }}=C_{\text {Trans }}+C_{\text {Process }}+C_{H l g}+C_{\text {strg }}$,

Where the $C_{p i}, C_{R e m}, C_{R e c}$ and $C_{L f}$ represent the life-cycle costs to produce new part $i$, remanufacture, recycle and land fill part $i$, respectively. The miscellaneous, transportation, reprocessing, handling and storage costs of the EOL part $i$ are represented by $C_{\text {Misc }}, C_{\text {Trans }}, C_{\text {Process }}, C_{H l g}$ and $C_{s t r g}$, respectively. The $V_{R e m}, V_{R e c}$ and $V_{L f}$ are the EOL values for remanufacturing, recycling and land filling of the EOL part $i$, individually. In order to reflect the environmental influence for selecting the EOL options of parts and subassemblies, Eqs. (5)-(8) were further elaborated as follows (Ghazalli \& Murata, 2011): 
$V_{\text {Rem }}=\left(C_{p i}+C_{\text {penv }}\right)-\left(C_{\text {Rem }}+C_{\text {EnvRem }}\right)-\left(C_{\text {Misc }}+C_{\text {EnvMisc }}\right)$,

$V_{\text {Rec }}=\left(C_{\text {Rec }}+C_{\text {EnvRec }}\right)-\left(C_{\text {Misc }}+C_{\text {EnvMisc }}\right)$,

$V_{L f}=-\left(C_{L f}+C_{E n v L f}\right)-\left(C_{M i s c}+C_{\text {EnvMisc }}\right)$,

$C_{\text {EnvMisc }}=C_{\text {EnvTrans }}$,

\subsubsection{Economical cost of new parts}

The economical cost of producing new parts is the cost of transforming a raw material into a finished product. It can be represented as follows:

$C_{p i}=C_{\text {material }}+C_{\text {manufacturing }}+C_{\text {operation }}$

where $C_{p i}$ is the economical cost of producing a new part $i, C_{\text {material }}$ is the material cost of part $i$ and $C_{\text {operation }}$ is the operational cost of part $i$.

The manufacturing cost includes the cost of manufacturing process, assembly, labour and overhead. Additionally, the operational cost comprises the cost of marketing, administration, distribution and so on (Kaebernick et al., 2002).

\subsubsection{Environmental cost of new parts}

The environmental cost of producing new parts encompasses the costs of energy consumed to form a raw material into a finished product. It can be represented as follows:

$C_{\text {penv }}=C_{\text {envmaterial }}+C_{\text {envmanufacturing, }}$

where $C_{\text {envmaterial }}$ is the environmental cost of a material to produce part $i$, and $C_{\text {envmanufacturing }}$ is the environmental costs to manufacture part $i$.

\subsubsection{Economical cost of remanufacturing parts}

The economical cost of remanufacturing parts consists of the costs of disassembly, sorting, cleaning, refurbishing, testing and reassembly. It can be represented as follows:

$C_{\text {Rem }}=\left(t_{\text {dis }}+t_{\text {sort }}+t_{\text {Clean }}+t_{\text {Ref }}+t_{\text {test }}+t_{\text {assy }}\right) \times \dot{C}_{L b}$

where $C_{\text {Rem }}$ is the remanufacturing cost, $t_{\text {dis }}, t_{\text {sort }}, t_{\text {Clean }}, t_{\text {Ref }}, t_{\text {test }}$ and $t_{\text {assy }}$ represent the time to disassemble, sort, clean, refurbish, test and reassemble of remanufactured part, respectively. $\dot{C}_{L b}$ is the labour cost.

\subsubsection{Environmental cost of remanufacturing parts}

The environmental cost of remanufacturing parts involves the costs of energy consumed for disassembling, sorting, cleaning, refurbishing, testing and reassembling the parts of the EOL product. It canbe represented as follows:

$C_{\text {EnvRem }}=E_{\text {Rem }} \times \dot{E}$,

where $E_{\mathrm{Rem}}$ is the total cost of energy used to remanufacturing part $i$, and the $\dot{E}$ is the energy consumption rate per unit, $\mathrm{kWh}$. 


\subsubsection{Economical cost of recycling parts}

The economical cost of recycling parts can be represented as follows:

$C_{R e c}=M_{R e c} \times w$,

where $M_{R e c}$ is the material cost per $\mathrm{kg}$, and $w$ is the material weight.

\subsubsection{Environmental cost of recycling}

The environmental cost of recycling parts cover the costs of energy consumed for disassembling, separating and shredding an EOL part. It can be represented as follows:

$C_{\text {EnvRec }}=C_{\text {RecE }} \times w$,

where $C_{\text {RecE }}$ is the environmental cost rate for recycling part $i$ per kg.

\subsubsection{Economical cost of land filling parts}

The economical cost of land filling parts can be represented as follows:

$C_{L f}=M_{L f} \times w$,

where $M_{L f}$ is the material cost per kg.

\subsubsection{Environmental cost of land filling part}

The environmental cost of land filling parts can be represented as follows:

$C_{E n v L f}=C_{L f E} \times w$,

where $C_{E n v L f}$ is the environmental cost rate for land filling part $i$ per kg.

\subsubsection{End-of-life profit and net present value assessment}

In this stage, the EOL profit and NPV are included for evaluating the end-of-life parts and subassemblies of the EOL product. The difference between EOL value and disassembly cost are used to find the most suitable and profitable EOL options. The EOL profit can be represented as follows:

$$
\begin{aligned}
& P_{E O L_{k}}=\left\{\begin{array}{lll}
V_{R e m}-C_{D i s} & \text { if } & k=\text { remanufacturing } \\
V_{R e c}-C_{D i s} & \text { if } & k=\text { recycling } \\
V_{L f}-C_{D i s} & \text { if } & k=\text { landfill }
\end{array}\right. \\
& C_{D i s}=t_{d i s} \times C_{l b}+C_{\text {tool }}+C_{\text {ohd }}, \\
& N P V_{E O L_{k}}=\frac{P_{E O L_{k}}}{(1+r)^{t}}
\end{aligned}
$$

where $C_{D i s}$ is the cost to disassemble part $i, t_{\text {dis }}$ is the labor time to disassemble part $i$ from an EOL product and $\dot{C}_{L b}$ is the labour rate per hour( $\left.\$ / \mathrm{hrs}\right) . C_{\text {tool }}$ and $C_{o h d}$ denote the cost of using tool(s) during disassembling part $i$ and is the overhead cost of disassembled part $i$, individually.

$P_{E O L_{k}}$ and $N P V_{E O L_{k}}$ are the EOL profits and net present values of remanufacturing, recycling or land filling $(k)$ options, respectively. In addition, $r$ and $t$ present the discount rate and end-of-life time of 
the EOL product, respectively. If the $V_{R e m}$, is higher than disassembly cost, the $N P V_{E O L_{R e m}}$ is more than zero, and the EOL profits of remanufacturing is higher than that of the recycling and land fill, the remanufacturing will be consider as an EOL option. If $V_{\text {Rem }}$ is lower than disassembly cost, the part or subassemblies under consideration should be further evaluated for recycling or land filling.

\section{Result: Application of the numerical experiments}

We examined the effectiveness of TSP-GA in finding the feasible sequence through numerical experiments. The algorithms of TSP-GA are coded in MATLAB. In the first experiment, we utilized the data from Kongar and Gupta (2006) to verify the effectiveness of TSP-GA by comparing it with the conventional GA.

Table 3 shows a comparison of the optimal disassembly sequence between the proposed algorithms of TSP-GA and the traditional GA method (Kongar \& Gupta 2006).

Table 3

Comparison between proposed algorithm and established method

\begin{tabular}{lll}
\hline & Proposed method & Established GA method \\
\hline $\begin{array}{l}\text { Disassembly } \\
\text { sequence }\end{array}$ & $2 \rightarrow 1 \rightarrow 0 \rightarrow 7 \rightarrow 6 \rightarrow 9 \rightarrow 3 \rightarrow 5 \rightarrow 4$ & $2 \rightarrow 1 \rightarrow 0 \rightarrow 7 \rightarrow 3 \rightarrow 6 \rightarrow 9 \rightarrow 5 \rightarrow 8 \rightarrow 4$ \\
Total time (sec) & 21 & 29 \\
\hline
\end{tabular}

In the second experiment, we used the body of an office deskphone as an example of a case study to investigate the effectiveness of TSP-GA by comparing it with traditional TSP(Moonet al. 2002). The diagram of the disassembly precedence constraint of a desk phone body is shown in Fig. 5 The TSPGA is repeated for 500 generations.

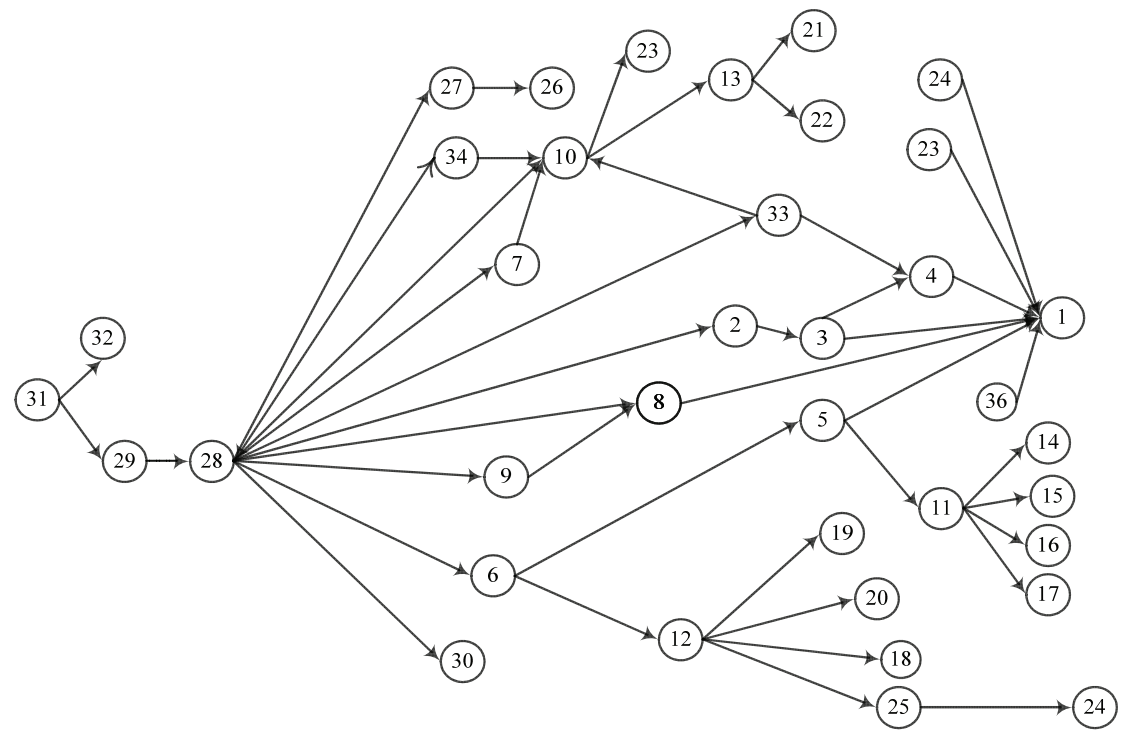

Fig.5. Disassembly precedence diagram of a desk phone body 
Table 4 shows a comparison of the optimal disassembly sequence between the proposed algorithms of TSP-GA and the established TSP method (Moon et al., 2002).

Table 4

Test result of disassembly sequence of TSP-GA.

\begin{tabular}{lll}
\hline & Proposed method & Established TSP method \\
\hline Disassembly & $31 \rightarrow 29 \rightarrow 30 \rightarrow 28 \rightarrow 34 \rightarrow 8 \rightarrow 33 \rightarrow 9 \rightarrow 27$ & $31 \rightarrow 29 \rightarrow 32 \rightarrow 28 \rightarrow 33 \rightarrow 2 \rightarrow 7 \rightarrow 3 \rightarrow 8$ \\
sequence & $\rightarrow 6 \rightarrow 5 \rightarrow 7 \rightarrow 2 \rightarrow 26 \rightarrow 12 \rightarrow 25 \rightarrow 3 \rightarrow 10 \rightarrow$ & $\rightarrow 27 \rightarrow 26 \rightarrow 4 \rightarrow 6 \rightarrow 5 \rightarrow 34 \rightarrow 10 \rightarrow 13 \rightarrow$ \\
& $23 \rightarrow 13 \rightarrow 4 \rightarrow 24 \rightarrow 11$ & $23 \rightarrow 12 \rightarrow 25 \rightarrow 11 \rightarrow 16 \rightarrow 15 \rightarrow 14 \rightarrow 17$ \\
& $\rightarrow 17 \rightarrow 14 \rightarrow 16 \rightarrow 20 \rightarrow 21 \rightarrow 18 \rightarrow 19 \rightarrow 15$ & $\rightarrow 18 \rightarrow 22 \rightarrow 19 \rightarrow 20 \rightarrow 21 \rightarrow 35 \rightarrow 36 \rightarrow 9$ \\
& $\rightarrow 22 \rightarrow 35 \rightarrow 36 \rightarrow 1 \rightarrow 32$ & $\rightarrow 24 \rightarrow 1 \rightarrow 30$ \\
Total time $(\mathrm{sec})$ & 150.26 & 151.87 \\
\hline
\end{tabular}

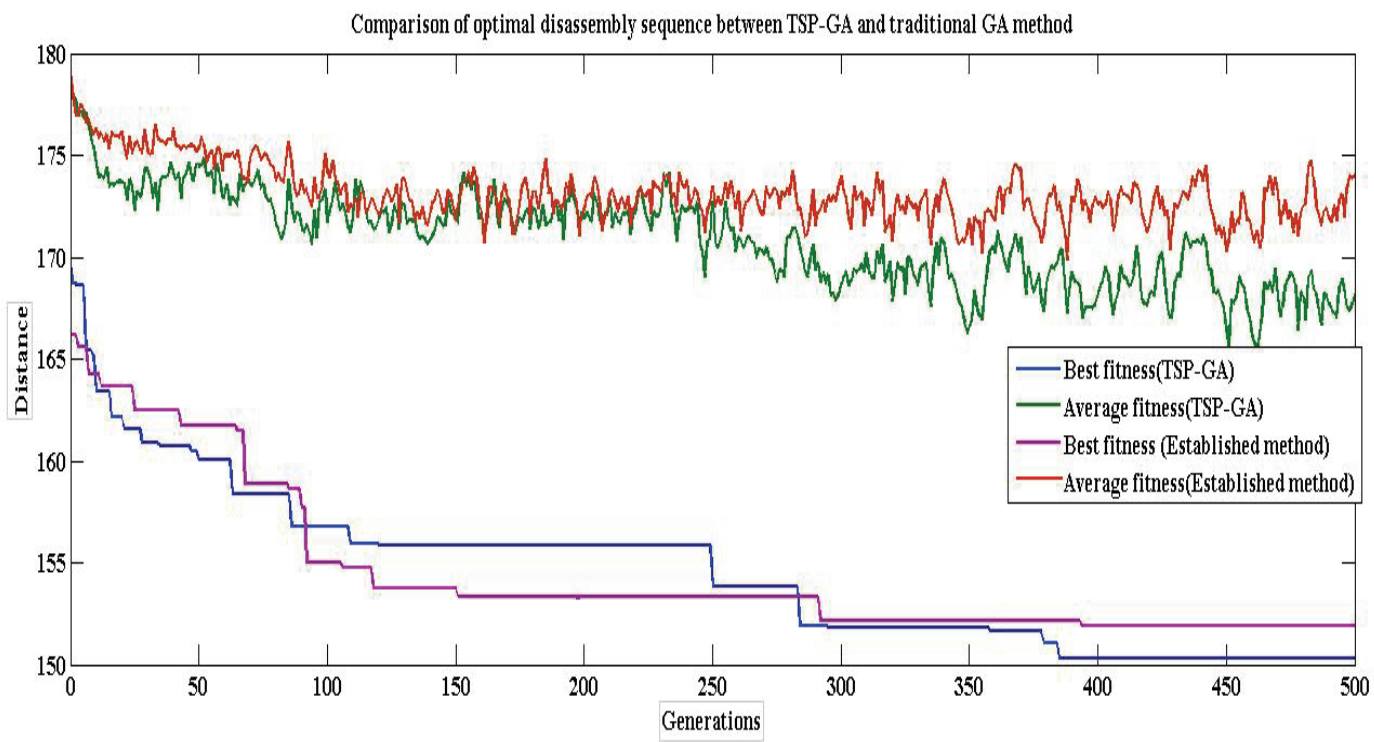

Fig.6. Convergence feature in TSP

Fig. 6 shows the integrated TSP-GA algorithm can derive the optimal disassembly sequence which convergence with the increase of generation. After the optimal disassembly sequence has been determined, the EOL profit and NPV for every part and subassembly is evaluated. Table 5 shows the comparison of EOL profits, NPV and the recommended EOL options between the proposed method and the traditional methods (Johnson 2002, Ghazalli \& Murata 2011). The agreement of the result obtained from the proposed cost model with that from previous conventional methods validates our cost model. The costs of procurement and remanufacturing are based on the percentages of the remanufacturing cost distribution shown in Fig.7 (Sudin \& Brass, 2005). After discussing with a manufacturer, a discount rate, $r$, is set to $15 \%$. The end-of-life time, $t$, is defined as a duration from which the product is purchased until it no longer meets the original functions. In the case of a desk phone body, we obtained the $t$ of the telephone from Rose (2000) which is 5 years. As some of the parameters are beyond our control, we used the following assumption taken from previous literatures.

1. The environmental cost of a remanufactured part was assumed to be $1 \%$ of the cost of a remanufactured part (Kaebernick et al., 2002).

2. The environmental cost rate for $C_{\text {Envtrans }}$ was assumed to be $\$ 0.146$ per ton-kilometre $(\$ / \mathrm{km})$ (Anityasari, 2008). 
3. The labour and landfill costs were set to $\$ 20$ per hour and $\$ 0.044$ per kilogram, respectively (Johnson, 2002).

4. The recycling rate of steel, lead (plumbum) and rubber were set to $\$ 0.90, \$ 0.211$ and $\$$ 0.01 per kilogram, individually (Johnson, 2002; Anityasari 2008). Additionally, the recycling rate for $\mathrm{ABS}, \mathrm{PVC}$ and $\mathrm{PP}$ were set to $\$ 0.11$ per kilogram each (Johnson, 2002).

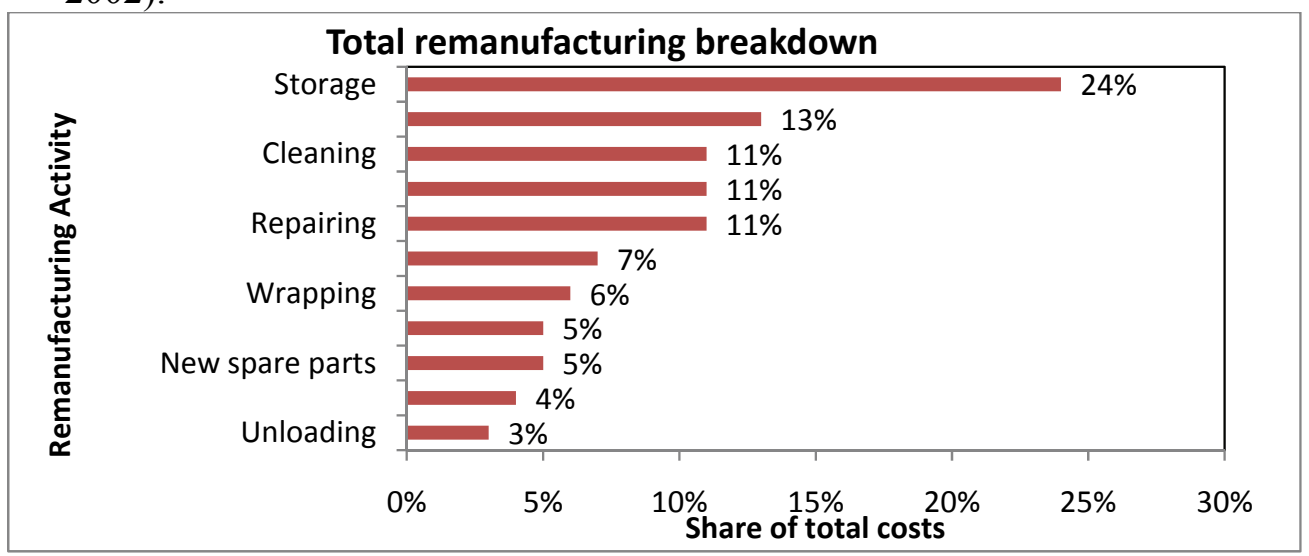

Fig.7. Cost distribution for product being remanufactured at Electrolux remanufacturing center in Montola (Sudin \& Bras 2005)

\section{Discussion}

Comparing the generated optimal disassembly sequence between TSP-GA and established method, the total time of TSP-GA is better than the conventional GA (Table 3). The first four-disassembly sequence between TSP-GA and established method are in the same order. However, the result demonstrates that the disassembly sequences from the fifth to tenth sequence of TSP-GA are contradictory to the conventional GA. One possible explanation is that the TS used in TSP-GA repaired the infeasible route of disassembly sequences before it goes to the next steps. On the other hand, the conventional GA used the heuristic method in finding the feasible chromosomes, which required complex procedures(Kongar and Gupta, 2006). As a result, TSP-GA presents the feasible disassembly sequence faster than the conventional GA.

Fig.5 shows that the path of the disassembly precedence relation is complete if the path visits all the tasks, and it is feasible if it does not violate the precedence constraint. As shown in Fig. 5, Task 31 is the non-predecessor task. As explained in the sub-section 2.2.2.1 through 2.2.2.3, this task must be disassembled first; then the new non-predecessor tasks are the Task 32 and Task 29. Therefore, either the Task 32 or Task 29 should be disassembled first. It shows that the Task 29 is the first to be found (see Table 4). Therefore, the following disassembly task is the Task 29 (refer to sub-section 2.2.2.2) and the redefined available set consists of the Task 28 and Task 32. This procedure continues until it thoroughly finishes disassembling all the vertices. Our finding shows that, the TS can be used to obtain all the feasible paths in the disassembly precedence diagram of the desk phone body (Fig. 5). Additionally, it also suggests that the TSP-GA should utilize the TS to generate all the feasible chromosomes at the representation stage.

Table 4 compares the proposed TSP-GA method with the conventional TSP method. The proposed method corresponds well with the conventional method. However, the sequence of chromosomes of the TSP-GA differs from the conventional TSP. A possible justification is that, in the TSP-GA, the TS utilized the disassembly precedence relationship (see Fig. 5) to generate the feasible chromosome. Additionally, at this stage, the TS also identified infeasible chromosomes and eliminated them. However, the conventional TSP indirectly generated the feasible chromosomes by comparing the priority among the tasks in the available set, which required very complex procedures. Additionally, the chances of chromosome to achieve the optimal solution is slightly low and time consuming as the 
chromosomes have to compare the highest priority among the tasks in the available set. Hence, the proposed TSP-GA shows more optimal disassembly sequence than the traditional TSP.

\section{Table 5}

EOL profits, net present value and EOL options of a deskphone body

\begin{tabular}{|c|c|c|c|c|c|c|c|c|c|c|}
\hline \multirow[b]{2}{*}{ No } & \multirow[b]{2}{*}{ Part name } & \multicolumn{3}{|c|}{ EOL profits } & \multicolumn{3}{|c|}{ Net present values } & \multicolumn{3}{|c|}{ EOL options } \\
\hline & & Rem & Rec & Lan & $\mathrm{NPV}_{\text {Rem }}$ & $\mathrm{NPV}_{\mathrm{Rec}}$ & $\mathrm{NPV}_{\text {Lf }}$ & EOL & AHP-CBR & $\begin{array}{l}\text { Tra } \\
\text { d }\end{array}$ \\
\hline 1 & Upper plastic casing & 12.953 & -0.255 & -0.238 & 6.440 & -0.127 & -0.118 & Rem & Rem & Rem \\
\hline 2 & Speaker foam & 0.262 & -0.005 & -0.224 & 0.130 & -0.003 & -0.112 & Rem & Rem & Rem \\
\hline 3 & 4 screws & -0.271 & 0.001 & -0.245 & -0.135 & 0.001 & -0.122 & $\operatorname{Rec}$ & $\operatorname{Rec}$ & $\operatorname{Rec}$ \\
\hline 4 & Speaker & 2.544 & 0.040 & -0.228 & 1.265 & 0.020 & -0.113 & Rem & Rem & Rem \\
\hline 5 & Plastic plate & 0.741 & -0.009 & -0.232 & 0.368 & -0.004 & -0.115 & Rem & Rem & $\operatorname{Rec}$ \\
\hline 6 & PolyFlex circuit board 1 & 3.955 & -0.005 & -0.260 & 1.967 & -0.002 & -0.129 & Rem & Rem & Rem \\
\hline 7 & PolyFlex circuit board2 & 8.516 & -0.005 & -0.285 & 4.234 & -0.002 & -0.142 & Rem & N/A & N/A \\
\hline 8 & screws & -0.701 & 0.001 & -0.241 & -0.348 & 0.001 & -0.120 & $\operatorname{Rec}$ & N/A & $\mathrm{N} / \mathrm{A}$ \\
\hline 9 & LCD panel\#1 & 5.244 & -0.003 & -0.237 & 2.607 & -0.001 & -0.118 & Rem & Rem & Rem \\
\hline 10 & Main circuit board & 6.290 & -0.007 & -0.238 & 3.127 & -0.003 & -0.118 & Rem & Rem & Rem \\
\hline 11 & Elastometer key pad \#1 & 1.210 & -0.005 & -0.226 & 0.601 & -0.002 & -0.112 & Rem & Rem & Rem \\
\hline 12 & Elastometer key pad \#2 & 0.866 & -0.003 & -0.226 & 0.430 & -0.001 & -0.112 & Rem & Rem & Rem \\
\hline 13 & Elastometer key pad \#3 & 0.852 & -0.002 & -0.226 & 0.424 & -0.001 & -0.112 & Rem & Rem & Rem \\
\hline 14 & Shift button & -0.070 & -0.001 & -0.224 & -0.035 & 0.000 & -0.111 & $\operatorname{Rec}$ & Lf & $\operatorname{Rec}$ \\
\hline 15 & 12 memory keys & 0.597 & -0.006 & -0.225 & 0.297 & -0.003 & -0.112 & Rem & Rem & $\operatorname{Rec}$ \\
\hline 16 & 3 display buttons & 0.039 & -0.002 & -0.224 & 0.019 & -0.001 & -0.111 & Rec & $\mathrm{Rec}$ & Rec \\
\hline 17 & Feature button & -0.020 & -0.002 & -0.224 & -0.010 & -0.001 & -0.111 & $\operatorname{Rec}$ & $\operatorname{Rec}$ & Rec \\
\hline 18 & Release button & -0.045 & -0.001 & -0.224 & -0.022 & -0.001 & -0.111 & $\operatorname{Rec}$ & Rec & Rec \\
\hline 19 & Hold button & 0.155 & -0.001 & -0.224 & 0.077 & -0.001 & -0.111 & $\operatorname{Rec}$ & Rem & Rem \\
\hline 20 & 12 dial keys & 0.556 & -0.008 & -0.224 & 0.276 & -0.004 & -0.112 & Rem & Rem & Rem \\
\hline 21 & 12 line keys & 0.494 & -0.011 & -0.225 & 0.245 & -0.005 & -0.112 & Rem & Rem & $\operatorname{Rec}$ \\
\hline 22 & Volume control & -0.030 & -0.001 & -0.224 & -0.015 & -0.001 & -0.111 & $\operatorname{Rec}$ & $\operatorname{Rec}$ & Rec \\
\hline 23 & Display cover - LCD\#1 & 0.169 & -0.004 & -0.274 & 0.084 & -0.002 & -0.136 & Rem & Rem & Rem \\
\hline 24 & Display cover - LCD\#2 & 0.179 & -0.003 & -0.274 & 0.089 & -0.002 & -0.136 & Rem & Rem & Rem \\
\hline 25 & LCD panel \# 2 & 0.629 & 0.000 & -0.236 & 0.312 & 0.000 & -0.117 & Rem & Lf & Lf \\
\hline 26 & Hang up lever & 1.024 & -0.005 & -0.256 & 0.509 & -0.002 & -0.128 & Rem & Rem & Rem \\
\hline 27 & Metal clip & 0.082 & 0.001 & -0.254 & 0.041 & 0.000 & -0.126 & Rem & Rem & Rem \\
\hline 28 & Lower casing & 14.998 & -0.364 & -0.243 & 7.457 & -0.181 & -0.121 & Rem & Rem & Rem \\
\hline 29 & Lower casing screws & 0.750 & 0.002 & -0.245 & 0.373 & 0.001 & -0.122 & $\operatorname{Rec}$ & N/A & N/A \\
\hline 30 & 2 rubber pads & 0.208 & -0.001 & -0.260 & 0.103 & 0.000 & -0.129 & $\operatorname{Rec}$ & Rem & Rem \\
\hline 31 & Plastic stand & 7.873 & -0.164 & -0.271 & 3.914 & -0.081 & -0.135 & Rem & Rem & Rem \\
\hline 32 & 2 rubber pads & 0.208 & -0.001 & -0.260 & 0.103 & 0.000 & -0.129 & $\operatorname{Rec}$ & Rem & Rem \\
\hline 33 & PCB-Speaker Wire & 1.047 & 0.000 & -0.256 & 0.520 & 0.000 & -0.127 & Rec & N/A & N/A \\
\hline 34 & PCB-Microphone wire & 1.047 & 0.000 & -0.256 & 0.520 & 0.000 & -0.127 & Rec & N/A & N/A \\
\hline 35 & Microphone & 0.270 & 0.000 & -0.221 & 0.134 & 0.000 & -0.110 & $\operatorname{Rec}$ & N/A & N/A \\
\hline 36 & Microphone Cover & 1.084 & 0.000 & -0.226 & 0.539 & 0.000 & -0.112 & Rem & $\mathrm{N} / \mathrm{A}$ & N/A \\
\hline
\end{tabular}

Rem: Remanufacturing, Rec: Recycling, Lan: Landfill, EOL: EOL profit method, AHP-CBR: AHP-CBR method, Trad: Traditional method

As shown in Fig. 6, our results demonstrate that the proposed algorithm starts with high distance of fitness at the initial generation. It gradually decreased when it reached the generations of 380 . After 380 generations, the distance of fitness was stabilized to 150.26 seconds. On the other hand, the traditional TSP method started with a lower distance of fitness than the TSP-GA. The distance of fitness rapidly decreased until 100 generations. After 100 generations, it gradually decreased, and at the generation of 390 , it generated an optimal disassembly sequence with a fitness of 151.87 .The average of fitness between the proposed TSP-GA and the traditional TSP indicate no significance differences until 250 generations. However, after 250 generations, the TSP-GA and traditional TSP show a significance difference of average fitness. A possible explanation is that the TSP-GA maintains the genetic characteristics by selecting the disassembly tasks according to the initial position of chromosome. Additionally, the TSP-GA directly adopts the sequence of disassembly tasks as chromosomes, the advantages of which enable the changes of sequence to follow directly the genetic algorithm for a better solution.

Additionally, Moon et al. (2002) adopted a traditional TSP for generating an optimal sequence. Our approach and the traditional TSP are different in that all chromosomes were repaired to generate a 
feasible chromosome at the initial stage. In the traditional TSP, regardless of the feasibility, all chromosomes were used in the step of fitness evaluation, crossover and mutation. The chromosomes were repaired after the infeasible solution was found, which required complex procedures. Thus, the TSP-GA eliminated the iteration time to check and find the feasible chromosomes.

Another objective in this study is to find the most profitable revenue EOL options of parts and subassemblies of the EOL product. From the holistic point of view, the EOL profit of remanufacturing outperformed others (Table 5). This result indicates that it is more profitable to remanufacture these parts than to recycle or landfill from economical, environmental and disassembly perspectives. The EOL profit for landfill is always in deficit. For screws, display buttons, feature buttons and release button, the EOL profit of recycling is better than that of remanufacturing. In other words, the cost to disassemble these parts for remanufacturing and landfill is more expensive than that of recycling them. Additionally, the NPV of these parts shows deficits if remanufacturing process is selected as EOL options.

As shown in Table 5, the NPV of remanufacturing outclassed that of recycling and landfill. All the parts and subassemblies of the desk phone body except the screws (part no. 3 and 8), shift button, feature button, release button, and volume control button, show that they are worthy of remanufacture rather than recycle or landfill. On the contrary, the NPV of remanufacturing for parts such as the screws (part no. 3 and 8), shift button, feature button, release button, and volume control button, show deficits. Additionally, we found that these parts express profit if the EOL option is recycling. The NPV will display insufficiencies if the EOL option of the parts and subassemblies of the desk phone body is landfill.

Our results demonstrated a good correspondence with the previous studies (Johnson, 2002; Ghazalli \& Murata 2011; Table 5). Our finding, however, shows that EOL options of parts such as the shift button, and LCD panel\#2 are contradictory to the cost model of AHP-CBR method (Ghazalli \& Murata, 2011) and the conventional method (Johnson, 2002) and it is recommended to disregard the shift button. It seems that the manufacturer earns more profits by dumping these parts rather than remanufacturing or recycling them. As for LCD panel \#2, our approach shows better EOL option than AHP-CBR cost model and the traditional method. One possible explanation is that the cost to disassemble this part for remanufacturing is lower than that for recycling and land filling. Additionally, NPV of this part demonstrates that it is better to remanufacture these parts rather than recycling or dumping them.

The selection of EOL alternatives in our integrated cost models is very homogeneous to the traditional method (Johnson, 2002) and the AHP-CBR cost model (Ghazalli \& Murata, 2011). A possible explanation is that we apply a similar strategy to these two methods. The differences between our proposed cost model from these two methods is that we consider the selection of EOL options from the EOL profits and NPV viewpoint, which represents economic, environment and disassembly perspectives. On the other hand, Johnson (2002) only considered an economical aspect for selecting EOL options. Additionally, Ghazalli and Murata (2011) integrated the economical and environmental cost factors for selecting EOL options. Our results demonstrate a good agreement with

Lee et al. (2002) and Kaebernick et al. (2002) who pointed out that economical and environmental cost are the main elements of determining the EOL alternatives of parts and subassemblies of a product. Additionally, as pointed out by Tang et al.(2004), NPV seems to act as a more efficient way of selecting the best EOL option of parts and subassemblies of an EOL product.

\section{Conclusion}

We extended the work of Ghazalli and Murata (2011) by introducing the TSP-GA to determine the optimal disassembly sequence of the EOL product. Based on this sequence, we conducted the 
evaluation of the EOL profits and NPV to find the best EOL options of parts and subassemblies of the EOL product. We examined the effectiveness of the proposed approach through the numerical experiments. The results showed that the TSP-GA corresponded well with the traditional TSP and GA methods. Additionally, the EOL profits and NPV showed a close correspondence with previous studies (Johnson, 2002; Ghazalli \& Murata, 2011). However, some additional issues should be addressed in future work.

Currently, the TSP-GA optimizes the disassembly sequence based on the disassembly time. Further studies should consider incorporating the environmental and economic trade-off within the TSP-GA. That will link to a multi-objective optimization through trade-off analysis between economic, environmental and disassembly factors. It would also be attractive to incorporate an ergonomic analysis such as motion analysis of the disassembly sequence into the evaluation system.

\section{Acknowledgement}

The first author would like to thank the Malaysia Ministry of Higher Education for financial support of education at Okayama University.

\section{References}

Anityasari, M. (2008). Reuse of industrial products - a technical and economic model for decision support. Dissertation(PhD).The University of New South Wales, Australia.

Ayres,R., Ferrer, G., \& Leynseele, T.V. (1997). Eco-efficiency, asset recovery and remanufacturing, European Management Journal, 15(5), 557-574.

Azab, A., Ziout, A., \& ElMaraghy, W. (2011). Modeling and optimization for disassembly planning. Jordan Journal of Mechanical and Industrial Engineering, 5(1),1-8.ISSN:1995-6665.

Das,S.K., Yeldarajiah,P., \& Narendra, R. (2000). An approach for estimating the end-of-life product disassembly effort and cost. International Journal of Production Research, 38(3), 657-673.

Dini, G., Falilli, F., \& Santochi, M. (2001). A disassembly planning software system for optimization of recycling process. Production Planning and Control, 12(1), 2-12.

ElSayed , A., Kongar, E., \& Gupta, S.M. (2010). A genetic algorithm approach to end-of-life disassembly sequencing for robotic disassembly. Proceedings of 2010 Northeast Decision Sciences Institute Conference, Alexandria, Virginia, 402-408, March 26-28.

Ghazalli, Z. \& Murata, A. (2011). Development of an AHP-CBR evaluation system for remanufacturing: End-of-life selection strategy. International Journal of Sustainable Engineering, $4(1), 2-15$.

Gungor, A., \& Gupta, S.M. (2001). Disassembly sequence plan generation using a branch-and-bound algorithm. International Journal of Production Research, 39(3),481-509.

Henrioud, J.-M, Relange, L., \& Perrard, C. (2003). Assembly sequences, assembly constraints, precedence graphs. Proceedings of the IEEE International Symposium on Assembly and Task Planning, 90-95.

Hui, W., Dong, X., \& Guanghong, D. (2008). A genetic algorithm for product disassembly sequence planning. Neurocomputing, (13-15), 2720-2726.

Ilgin, M.A., \& Gupta, S.M. (2010). Environmentally conscious manufacturing and product recovery: a review the state of art. Journal of Environmental Management, 91(3), 563-591.

Johnson, M.R. (2002). Evaluating remanufacturing and demanufacturing for extended producer responsibility \& sustainable product management. $\mathrm{PhD}$ dissertation.University of Windsor.

Kaebernick, H., Anityasari, M., \& Kara, S. (2002). A technical and economic model for end-of-life options of industrial product. International Journal of Environmental Sustainable Development, $1(2), 171-183$

Ke, C., \& Henrioud, J.-M. (1994). Systematic generation of assembly precedence graphs, Proceedings of IEEE International Conference on Robotics and Automation, Issue pt 2, 14761482, ISBN: 0818653329. 
Kongar, E., \& Gupta, S.M. (2006). Disassembly sequencing using genetic algorithm. International Journal of Advance Manufacturing Technology, 30, 497-506.

Krikke, H.R., Harten, A.V., \& Schuur, P.C. (1998). On a medium term product recovery and disposal strategy for durable assembly products. International Journal of Production Research, 36 (1), 111139.

Kusiak, A., \& Finke, G. (1987). Modeling and solving the flexible forging module scheduling problem. Engineering Optimization, 12(1), 61-68.

Lambert, A.D.J., \& Gupta, S.M. (2005). Disassembly modeling for assembly, maintenance, reuse and recycling.Florida: CRC Press.

Lee, S.G., Lye, S.W., \& Khoo. M.S. (2001). A multi-objective methodology for evaluating product end-of-life options and disassembly, International Journal of Advanced Manufacturing Technology, 18(2), 553-575.

Li, J.R., Khoo,L.P., \& Tor, S.B. (2005). An object-oriented intelligent disassembly sequence planner for maintenance.Computers in Industry, 699-718.

McGovern, S.M., \& Gupta, S.M. (2007). A balancing method and genetic algorithm for disassembly line balancing. European Journal of Operation Research, 179(3), 483-496.

Moon, C., Kim, J., Choi, G., \& Seo, Y. (2002). An efficient genetic algorithm for traveling salesman problem with precedence constraints. European Journal of Operational Research, 140, 606-617.

Penev, K.D. and De Ron, A. J. (1996). Determination of a disassembly strategy. International Journal of Production Research, 34(2), 495-506.

Renaud,J., Boctor, F.F., \& Ouenniche, J. (2000). A heuristic for the pickup and delivery traveling salesman problem. Computer \& Operations Research, 27(9), 905-916.

Ritchey,J.R., Mahmoodi, F., Frascatore, M.R., \& Zander, A.K. (2005). A framework to assess the economic viability of remanufacturing. International Journal of Industrial Engineering: Theory Applications and Practice, 12(1), 89-100.

Rose, C.M. (2000). Design for environment: a method for formulating product end-of-life strategies. Dissertation(PhD).Stanford University, Stanford, USA.

Seo, K.-K, Park, J.,-H., \& Jang, D.-S. (2001). Optimal disassembly sequence using genetic algorithm considering economic and environmental aspect. International Journal of Advanced Manufacturing Technology, 18, 371-380.

Srinivasan,H., \& Gadh, R. (1998). A geometric algorithm for single selective disassembly using the wave propagation abstraction, CAD Computer Aided Design, 30(8), 603-613.

Staikos, T., \& Rahimifard, S. (2007). An end-of-life decision support tool for product recovery considerations in footwear industry, International Journal of Computer Integrated Manufacturing, 20(6), 602-615.

Sundin, E., \& Bras, B. (2005). Making functional sales environmentally and economically beneficial through product remanufacturing, Journal of Cleaner Production, 13(9), 913-925.

Tan, A., \& Kumar, A. (2008). A decision making model to maximize the value of reverse logistics in the computer industry, International Journal of Logistics Systems and Management (IJLSM), 4(3), 297-312.

Tang,O., Grubbström, R.W. \& Zanon, S. (2004). Economic evaluation of disassembly process in remanufacturing system. International Journal of Production Research, 36 (1), 111-139.

Xanthopolus, E., \& Iakouvou, A. (2009). On the optimal design of the disassembly and recovery processes. Waste Management, 29, 1702-1711.

Xirouchakis, P. \& Kritis, D. (1997). Petri net modeling for disassembly process planning, American Society of Mechanical Engineers, Design Engineering Division (Publication) DE, 94, 255-262, ISSN: 15214613.

Zussman, E., Kriwet, A., \& Seliger, G. (1994). Disassembly-oriented assessment methodology to support design for recycling, CIRP Annals-Manufacturing Technology, 43(1), 9-14, ISSN: 00078506,DOI: 10.1016/S0007-8506(07)62152-0. 